\title{
POSTNATAL GROWTH OF THE BRONCHI AND BRONCHIOLES
}

\author{
BY \\ R. E. CUDMORE, J. L. EMERY and A. MITHAL \\ From the Department of Pathology, The Children's Hospital, Sheffield
}

(RECEIVED FOR PUBLICATION APRIL 19, 1962)

There is no question that the lung of the adult is a more complex structure and contains a considerably larger number of alveoli than that of the newborn child, but the method of growth of the lung is still incompletely understood, particularly with regard to the sites of postnatal proliferation and maximal growth.

While the study of minute fragments of the lung is relatively easy, that of the detailed structure of the whole of a lobe is surprisingly difficult. Miller (1947) maintained that the only way to study lung development was by serial sectioning and reconstruction, but even he confined himself mainly to small segments of the lung and isolated parts of the bronchial tree. In a study of the development of the bronchi, Hayward and Reid (1952), introduced the concept of studying axial pathways and combined gross dissection with serial sections in an attempt to determine the number of generations of bronchi in adult lungs. Recently Bucher and Reid (1961), applied this same method to the lungs of foetuses and came to the conclusion that the branching of the main bronchi of the lung occurred chiefly during the first 14 weeks of intrauterine life to reach a maximum between the 15th and 20th week. Following this there appeared, surprisingly, to be a slight reduction in the number of generations of bronchi.

We have previously shown (Emery and Mithal, 1960) that there is a great increase in the number of alveoli in the terminal respiratory unit following birth, but it has not been possible to determine if and how new terminal respiratory units develop. Since the introduction of resins with delayed and controlled solidification times (Tompsett, 1952), it is now possible to obtain excellent casts of the respiratory tree including alveoli. In the present study we have attempted to assess the numbers of generations of bronchi along the axial paths of the right upper lobe of the lung at different ages and have attempted to determine where maximum growth occurs within this system.

\section{Material and Method}

Fresh lungs were used from autopsies. Only lungs which appeared to be normal were used and casts were made of the right upper lobes of these lungs as described by Tompsett (1952) using Marco resin 26C. Gelatine was not used first so that filling of the terminal alveoli was achieved in most cases and the lungs were not pruned. Casts were made from about 50 lungs, but only perfect casts, 20 in number, having well-defined axial pathways were used. They ranged in age from 37 weeks' gestation to 73 years after birth. The casts were trimmed, leaving the axial pathways and terminal bunches of alveoli. Maximal counts only were recorded. The segmental bronchus was regarded as the first generation, and counting was continued until the point of apparent division of ducts into small groups of alveoli. This latter point was not at all easy to determine and careful dissection under the plate microscope was necessary. In general the end point in counting generations probably corresponds to the millimetre pattern described by Reid and Simon (1958). The appearance of a partly trimmed cast and also of the terminal portion of a trimmed cast are shown in Figs. 1 and 2.

The axial counts from the upper right lobes of four stillborn children of 37 weeks' gestation were $15,14,16,14,16 ; 18,20,16,15,14 ; 17,18 ; 17,18$, $19,18,14,15$; the maximal counts being 16, 20, 18, 19. The axial counts from four stillborn children born at 40 weeks' gestation were as follows: $21,19,20,21 ; 15,14,17,17,15,13,13 ; 18,20,21$, $22,21,20 ; 18,19,1918$; the maximal counts were $21,17,22$ and 19. One cast from a child dying two weeks after birth following $\mathbf{4 0}$ weeks' gestation was $21,22,20,19,18,20$ and one child aged 3 weeks, following full-term gestation, 18, 19, 15, 16, 17 . A cast from a child of 18 months was $17,18,18$, $19,16,17,16$; from a child of $2 \frac{1}{2}$ years $19,19,19$, 17, 18, 18, 18; from one child of 3 years $18,20,18$, $17,19,17$; and from a child of 12 years $17,16,18,19$. From adult lungs, one aged 22 years 17, 18; one 


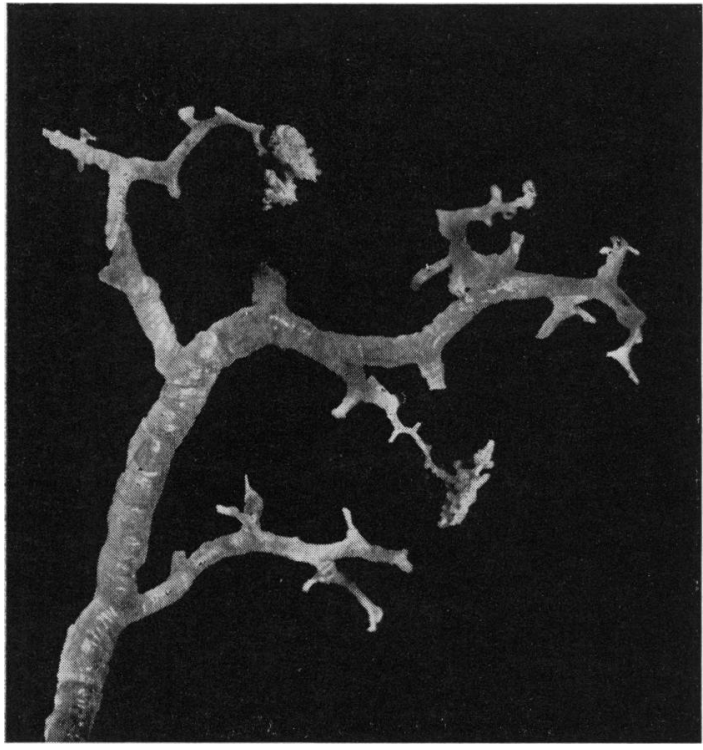

Fig. 1.-A cast which has been partly trimmed to show the branching to the alveoli along lateral branches of the axial tree.

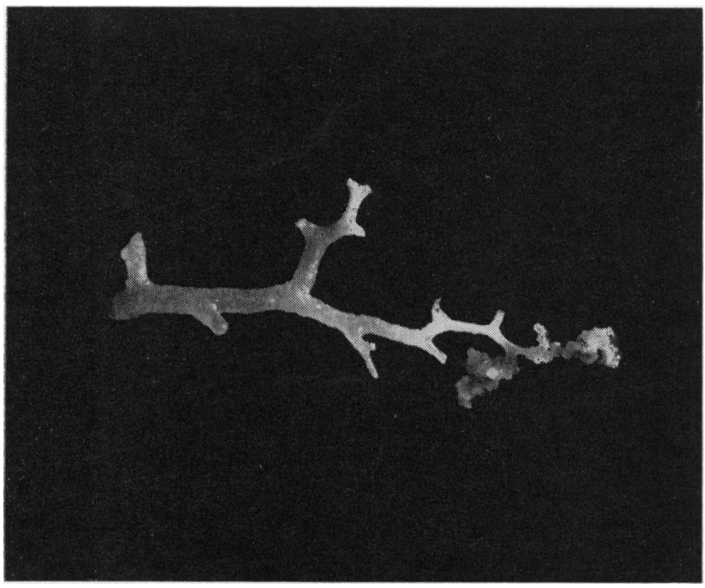

FIG. 2.-A trimmed cast showing the tip of an axial pathway.

of 29 years $18,17,17,17,17,18$; one of 58 years $18,17,19$; one of 73 years $18,17,17$; and two further casts from adults aged between 50 and 60 years, gave counts of $16,16,16,17,17$. As the maximum number is the most significant in any lobe the maximum numbers for each lung examined are shown in Fig. 3. The greatest variation appeared to occur around birth. There does not seem to be any increase in the number of bronchial generations between birth, adolescence and adult maturity.

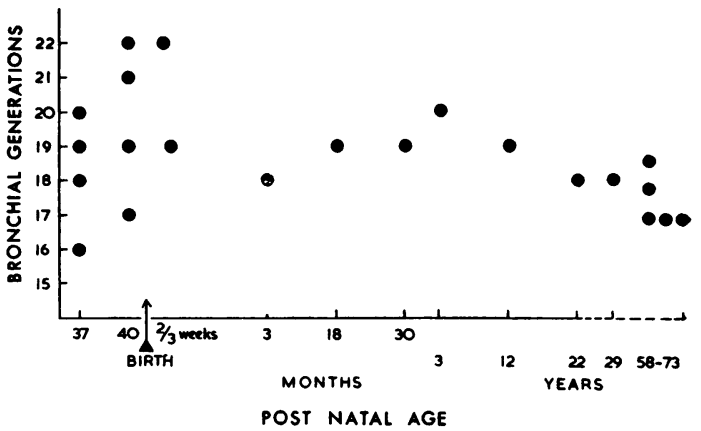

FIG. 3.-Showing the maximum number of bronchial generations in the right upper lobe of casts from lungs of patients of different ages.

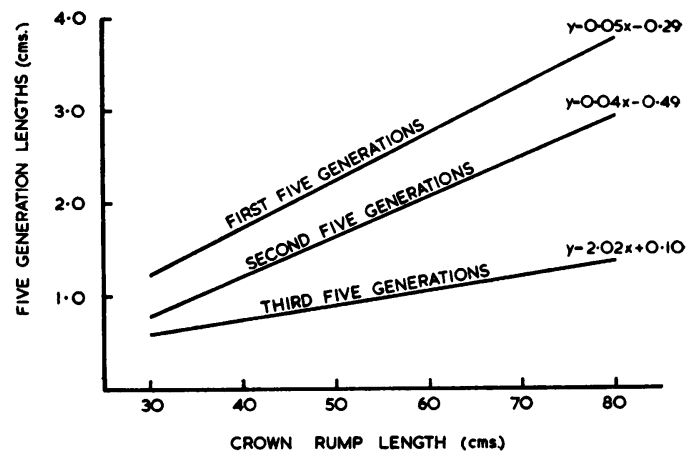

FIG. 4.-Showing regression lines of the lengths of the proximal, middle and distal segments of the axial bronchi in the right upper lobe related to crown rump length.

An attempt was made to determine the length of the bronchi between generations in different parts of the tree, but owing to the considerable variability of these measurements it was found best to divide the whole axial pathway measuring four or five generations together. Where five segments could be measured in a direct line, this was done with fine calipers or under the plate microscope. If only four segments were in a direct line these were measured and the result multiplied by $5 / 4$. The average intersegmental length in the three parts-proximal, middle and distal of the axial pathway-were then compared.

The regression lines of the lengths of the segments related to the crown rump length of the individual case are shown in Fig. 4. All three sections grow in proportion to the crown rump length and thus the total length of the axial pathway increases proportionately to the length of the individual. The data were surveyed by Martin Cliffe of the Sheffield University Department of Statistics who found that the regression equation for the first 
five divisions was $y=0.05 x \ldots 0 \cdot 29$; for the second divisions $y=2 \cdot 02 x+0 \cdot 10$, where $x$ (the independent variable) is the crown rump length. He reports 'when three groups of five generations were considered separately, it was found that the lengths correlated significantly with the crown rump length. The first five generations group length exceeds the second group length, which in turn is larger than the third which explains the different constant terms found in the three regression equations. A statistical comparison of the regression lines slopes proves significant in that the rate of increase of growth decreases with distance from the stem.'

An attempt was also made to measure, in selected casts, the cross-sectional area of the bronchi in succeeding generations. Our measurements confirmed the work of Barnett (1957) with adult casts, that the cross-sectional area increases by a factor of approximately $1 \cdot 3$ for each succeeding generation of bronchi. Our results are not therefore published in detail.

\section{Discussion}

Bucher and Reid (1961) have produced evidence suggesting that the total number of bronchial generations is the same in the 25 -week-old foetus as in the adult. Their study was based on naked eye dissection followed by serial sectioning of the terminal segments of foetal and adult lungs. They found a possible diminution in the total number of bronchial tree branches during the later stage of intrauterine life. In foetal lungs the distinction between alveoli lined by cuboid epithelium and air passages with similar epithelium is not easy. Thus, the apparent fall in number during the last 15 or so weeks of intrauterine life is possibly an artefact, but the measurement at birth is not liable to this type of inaccuracy.

Broman (1923) studying the right middle lobe in man, found an increased number of generations of the respiratory tract before the respiratory bronchioli. Engel (1947) also suggests that there is an increase in branching after birth.

One of the major difficulties in assessing lungs is the very great variability in the branching, and thus terminal respiratory units may be reached at some parts of the lung, particularly those near the hilum, following only half a dozen or so generations. The method that we have adopted, which seems to be the only practical solution to this problem, is that used by Lynne Reid and her associates, i.e. counting the branches of the axial bronchi. This gives the maximal number of generations within any particular lobe of the lung.
To find, as we have done, that there does not appear to be any increase after birth in the number of branchings from this axial pathway before the alveolar type structure is reached, does not mean that there is no postnatal branching as this could well occur in the non-axial parts of the bronchial tree. But it is virtually impossible to count all of the side branches. We initially attempted this and failed. When we counted the number of generations of bronchi to random segments of the lobe, a wide variation was found, alveoli being reached in some parts after only seven or eight generations. In order to prove or disprove increased branching among the lateral air passages, it would be necessary to produce a distribution curve of the number of generations existing in the whole lobe and to see if the shape or mean point of the distribution curve altered after birth.

It is this mean point and distribution that we eventually wish to know. In the meantime, our own findings confirm those of Bucher and Reid (1961) and E. A. Boyden (personal communication) in that we have found no evidence for an increase in branching of the longitudinal axis of the right upper lobe from birth to maturity. If this is true for the whole bronchial tree of the lobe, it directs interest in development into the terminal respiratory unit.

Our studies in the length of the different intersegmental differences in the proximal, middle and distal parts of the axial paths, suggest that the bronchi grow proportionately to the body's crown rump length and that the proximal generation of bronchi grow at a greater rate than those in the periphery.

\section{Summary}

The number of generations in the axial pathways of the right upper lobe of the lung have been counted in casts made from full-term foetuses and from children and adults.

No apparent increase in the number of generations was found.

The intersegmental lengths of the bronchi were measured, and this suggests that all segments of the bronchial axial tract grow proportionately to the child's crown rump length, but that the rate of growth of the proximal segments is greater than those at the periphery.

Dr. R. Cudmore and Dr. A. Mithal have been working under a grant from the Medical Research Council.

We are also grateful for advice from Dr. Lynne Reid and statistical assistance from Mr. Martin Cliffe. Photographs are by Mr. Ronald Cousins. 


\section{REFERENCES}

Barnett, C. H. (1957). A note on the dimensions of the bronchial tree. Thorax, 12, 175.

Boyden, E. A. Personal communication, quoted by Bucher and Reid (1961).

Broman, I. (1923). Zur Kenntnis der Lungenentwicklung. Anat. Anz., 57, Ergänzungsheft, p. 83.

Bucher, U. and Reid, L. (1961). Development of the intrasegmental bronchial tree: The pattern of branching and development of cartilage at various stages of intra-uterine life. Thorax, 16, 207.

Emery, J. L. and Mithal, A. (1960). The number of alveoli in the terminal respiratory unit of man during late intrauterine life and childhood. Arch. Dis. Childh., 35, 544.

Engel, S. (1947). The Child's Lung. Arnold, London.

Hayward, J. and Reid, L. McA. (1952). Observations on the anatomy of the intrasegmental bronchial tree. Thorax, 7, 89.

Miller, W. S. (1947). The Lung, 2nd ed. Thomas, Springfield, Illinois.

Reid, L. and Simon, G. (1958). The peripheral pattern in the normal bronchogram and its relation to peripheral pulmonary anatomy. Thorax, 13, 103.

Tompsett, D. H. (1952). A new method for the preparation of bronchopulmonary casts. ibid., 7, 78. 